OPEN ACCESS

Edited by:

Changhui Li,

Peking University, China

Reviewed by:

Antonello Enrico Spinelli,

San Raffaele Scientific Institute, Italy

Ralph Peter Mason,

University of Texas Southwestern

Medical Center, United States

*Correspondence:

Sarah E. Bohndiek

seb53@cam.ac.uk

${ }^{\dagger}$ Present address:

Michal R. Tomaszewski, Translational Imaging Department, Merck \& Co. Inc., West Point, PA, United States

Specialty section:

This article was submitted to

Cancer Imaging and Image-directed Interventions,

a section of the journal

Frontiers in Oncology

Received: 28 October 2021 Accepted: 07 February 2022

Published: 03 March 2022

Citation:

Lefebvre TL, Brown E, Hacker L,

Else T, Oraiopoulou M-E

Tomaszewski MR, Jena $R$ and

Bohndiek SE (2022) The Potential

of Photoacoustic Imaging

in Radiation Oncology.

Front. Oncol. 12:803777.

doi: 10.3389/fonc.2022.803777

\section{The Potential of Photoacoustic Imaging in Radiation Oncology}

\author{
Thierry L. Lefebvre ${ }^{1,2}$, Emma Brown ${ }^{1,2}$, Lina Hacker ${ }^{1,2}$, Thomas Else ${ }^{1,2}$, \\ Mariam-Eleni Oraiopoulou ${ }^{1,2}$, Michal R. Tomaszewski ${ }^{3+}$, Rajesh Jena ${ }^{4}$ \\ and Sarah E. Bohndiek ${ }^{1,2 *}$
}

${ }^{1}$ Department of Physics, University of Cambridge, Cambridge, United Kingdom, ${ }^{2}$ Cancer Research UK Cambridge Institute, University of Cambridge, Cambridge, United Kingdom, ${ }^{3}$ Department of Cancer Physiology, H. Lee Moffitt Cancer Center and Research Institute, Tampa, FL, United States, ${ }^{4}$ Department of Oncology, University of Cambridge, Cambridge, United Kingdom

Radiotherapy is recognized globally as a mainstay of treatment in most solid tumors and is essential in both curative and palliative settings. Ionizing radiation is frequently combined with surgery, either preoperatively or postoperatively, and with systemic chemotherapy. Recent advances in imaging have enabled precise targeting of solid lesions yet substantial intratumoral heterogeneity means that treatment planning and monitoring remains a clinical challenge as therapy response can take weeks to manifest on conventional imaging and early indications of progression can be misleading. Photoacoustic imaging (PAl) is an emerging modality for molecular imaging of cancer, enabling non-invasive assessment of endogenous tissue chromophores with optical contrast at unprecedented spatio-temporal resolution. Preclinical studies in mouse models have shown that PAI could be used to assess response to radiotherapy and chemoradiotherapy based on changes in the tumor vascular architecture and blood oxygen saturation, which are closely linked to tumor hypoxia. Given the strong relationship between hypoxia and radioresistance, PAl assessment of the tumor microenvironment has the potential to be applied longitudinally during radiotherapy to detect resistance at much earlier timepoints than currently achieved by size measurements and tailor treatments based on tumor oxygen availability and vascular heterogeneity. Here, we review the current state-ofthe-art in PAl in the context of radiotherapy research. Based on these studies, we identify promising applications of PAI in radiation oncology and discuss the future potential and outstanding challenges in the development of translational PAI biomarkers of early response to radiotherapy.

Keywords: photoacoustic (optoacoustic) imaging, radiation oncology, radiotherapy, quantitative imaging biomarker, image guidance, translational research

\section{INTRODUCTION}

External X-ray beam radiotherapy (EBRT) is a common and effective treatment for many solid tumors, used as a standalone method or in combination with other treatments, from first line to palliative setting (1). Depending on tumor site and other risk factors, EBRT can be administered as a primary treatment, in the neo-adjuvant setting, to shrink the mass and improve resection success 
rates, or in adjuvant setting to prevent recurrence (2-4). In conventionally fractionated EBRT, small fractions of radiation dose are delivered to the tumor over several weeks and optimized to spare surrounding healthy tissue (1). Methods that afford higher precision in dose planning and delivery through imageguidance enable higher doses to be delivered in fewer fractions, while achieving similar healthy organ preservation $(2,5)$.

Response to EBRT is typically assessed by the response evaluation criteria in solid tumors (RECIST) (6) and its derivatives $(7,8)$ in clinical trials. Such size-based assessments do not account for spatial heterogeneity, can take weeks to manifest, and may be misleading, such as in "pseudoprogression" $(9,10)$. Similarly, radiation-induced adverse effects in healthy tissue are a key concern for patients undergoing EBRT, but the first clinical signs may take weeks or months to appear $(11,12)$. In recent decades, the paradigm of response assessment in EBRT has been slowly redefined $(10,13)$ by the use of molecular imaging in addition to widely used anatomical imaging $(14,15)$. Molecular imaging can also improve EBRT pipelines by better targeting metabolically active tumor volumes (16-18).

Radiobiological response is strongly influenced by hypoxia, or oxygen starvation, in solid tumors $(19,20)$. The radiation dose required to achieve a given biological effect is up to 3-fold higher in hypoxic than in normoxic conditions (21). Conventionally fractionated radiotherapy can partially mitigate this through inter-fraction reoxygenation. In hypofractionated courses, such as stereotactic body radiotherapy (SBRT) and stereotactic ablative radiotherapy (SABR), radioresistance associated with hypoxia has been shown to increase in preclinical and modeling studies (22-24), potentially as a result of the partial loss of reoxygenation and the induced vascular damage, subsequently leading to oxygen deprivation. Radiotherapy regimens delivered in shorter timeframes make the need for adequate tumor oxygenation even greater. Moreover, tumors often display substantial spatial and temporal heterogeneity in hypoxia (20, 25 ), yet our ability to account for this phenomenon in treatment planning and response monitoring is fundamentally limited.

Current molecular imaging modalities afford some insight into the spatial distribution of tumor hypoxia (26). For example, tracers such as fluoromisonidazole $\left({ }^{18} \mathrm{FMISO}\right)$ in positron emission tomography (PET) can map tumor hypoxia and adjust dose escalation $(16,17)$ and de-escalation (18) accordingly. PET-CT scans are not typically performed at multiple timepoints, however, because of isotope cost, scanning time, and additional radiation exposure that needs to be justified. Magnetic resonance imaging (MRI) has also shown potential as a non-ionizing modality for defining sub-volumes for escalation of radiation dose based on diffusion $(27,28)$ and perfusion $(29-31)$ biomarkers, or for predicting response with oxygen-sensitive MRI techniques using tissue and blood oxygen level dependent (TOLD/BOLD) signals with oxygen (32) or carbogen gas breathing challenge (33), reviewed elsewhere (34). Nonetheless, these methods have limited spatio-temporal resolution, comparatively long acquisition times, and may require exogenous contrast agents, with associated toxicity $(35,36)$. Furthermore, they have limited capability for deployment in conventional linear accelerator rooms, except through combined MR-linear accelerator systems $(37,38)$ or novel PET-linear accelerator systems $(39,40)$, which are currently limited by high cost and complexity (41).

Photoacoustic imaging (PAI) is a clinically emerging localized imaging modality that enables affordable, real-time interrogation of oxyhemoglobin $\left(\mathrm{HbO}_{2}\right)$ and deoxyhemoglobin $(\mathrm{Hb})$ in tumors at high spatio-temporal resolution $(42,43)$. Based on the absorption of non-ionizing optical radiation (44-48), and associated generation of acoustic waves, PAI systems are readily combined with ultrasound given their shared signal detection schemes and provide intrinsically multi-modal imaging to up to approximately 5 $\mathrm{cm}$ depth with current technologies (49). When using multiple wavelengths for imaging and applying spectral unmixing algorithms, PAI data can be used to resolve endogenous imaging biomarkers related to total hemoglobin $\left(\mathrm{THb}=\mathrm{Hb}+\mathrm{HbO}_{2}\right)$ and blood oxygen saturation $\left(\mathrm{sO}_{2}=\mathrm{HbO}_{2} / \mathrm{THb}\right) . \mathrm{sO}_{2}$ measured with PAI has been shown to correlate with tumor hypoxia, using ex vivo histology as the reference standard $(44,45,50)$, demonstrating the potential of PAI to provide surrogate non-invasive biomarkers of tissue hypoxia. With the introduction of exogenous contrast agents, it is also possible to directly report on tumor tissue $\mathrm{pO}_{2}$ (51). Moreover, PAI is scalable for non-invasive assessment of single capillaries and even red blood cells (52) and can also be used to extract information on blood flow (53). PAI has thus been proposed for application in superficial tumors to improve: radiation dose delivery and scheduling; patient stratification; and therapy response and radiation side effects monitoring. Here, we summarize the potential of PAI as a fast, portable and affordable tool for monitoring of key radiobiological processes across different length scales, with a particular focus on vascular changes in normal and tumor tissue in response to radiation.

\section{POTENTIAL USES OF PHOTOACOUSTIC IMAGING IN RADIOTHERAPY}

\section{Measuring and Monitoring Tumor Response to Radiotherapy With Photoacoustic Imaging}

Doses of ionizing radiation delivered in clinical EBRT can induce acute endothelial cell dysfunction, blood vessel disruption, and mitotic catastrophe resulting in apoptosis, which can lead to secondary tissue necrosis (54). When entering tissue, low-linear energy transfer (LET) ionizing radiation, such as X-rays used clinically at $\mathrm{MeV}$ energy levels, produces free radicals through the radiolysis of water. If oxygen is present, free radicals form highly reactive peroxyl radicals, which lead to DNA damage and subsequently, cell death (21). In the absence of oxygen, free radicals can be neutralized by interacting with hydrogen or by electron donation, consequently minimizing radiation damage (55). The permeability of capillaries is enhanced after dose delivery and platelet aggregation and microthrombus formation is induced. Altered perfusion can often result, which may in turn cause hypoxia and tumor necrosis, affecting the tumor cell kill of further 
radiation fractions (56). The timescales and the extent of the change in blood flow to the tumor and in tissue reoxygenation are dependent on many factors, including dose fractionation and have yet to be systematically studied. At SBRT and SABR regimes (>10 Gy/fraction), perfusion changes have been observed in some preclinical studies (57). For instance, in DCE-MRI derived perfusion measurements decreased $2 \mathrm{~h}$ post-20 Gy delivery in orthotopic brain tumors in rats (58) although they did not change significantly in subcutaneous lung tumors post-12 Gy (59).

PAI has been examined in the context of EBRT response assessment in preclinical cancer models. Tumor $\mathrm{sO}_{2}$ was demonstrated to be an early biomarker of EBRT response in patient-derived xenografts of $\mathrm{H} \& \mathrm{~N}$ cancer, with higher $\mathrm{sO}_{2}$ being predictive of response to single dose radiation delivery (Figure 1A) $(60,62,63)$. Interestingly, increased $\mathrm{THb}$ levels during fractionated EBRT were associated with better treatment outcomes (64) and tumors responding to radiation had decreased $\mathrm{THb}$ in the early days post-EBRT $(64,65)$, suggesting PAI can evaluate both tumor sensitivity and early treatment response. Importantly, a dose per fraction of $3 \mathrm{~Gy} /$ day was sufficient to cause a significant $\mathrm{sO}_{2}$ change as early as 3 days into the treatment course (64), rather than waiting weeks for changes in tumor size to manifest.

EBRT also has the potential to stimulate tumoral neovascularization, as a result of the radiation-induced acute inflammatory response shortly after dose delivery (66). Therefore, blood flow to the tumor and tissue oxygenation may be transiently increased in the few hours following a dose of EBRT, potentially leading to an increase in $\mathrm{sO}_{2}$ and $\mathrm{THb}$. After EBRT, Hysi et al. reported increased $\mathrm{sO}_{2}$ both 2 and $24 \mathrm{~h}$ after a single 8 Gy dose of radiation (67), in an SBRT-like regimen. Interestingly, this also correlated with an increase in the expression of the endothelial marker $\mathrm{CD} 31^{+}$area measured ex vivo, compared to non-treated controls (67). Days after the end of $\mathrm{EBRT}, \mathrm{sO}_{2}$ and $\mathrm{THb}$ were found to be decreased compared to pre-treatment scans $(64,65)$. PAI has previously shown that a similar pattern of changes following antiangiogenic treatment may be attributed to vascular normalization, suggesting this mechanism could be observed also in the context of ablative EBRT (68). Since reoxygenation may be crucial for the treatment effectiveness of SBRT fractions (23), PAI offers an opportunity to noninvasively and longitudinally evaluate the timing at which tumors reoxygenate to plan fraction deliveries then.

Dynamic contrast-enhanced (DCE)-PAI has also been assessed as a marker of radiation response (63) using exogenous indocyanine green (ICG) to detect perfusion $(44,69)$. DCE-PAI was achieved with perfusion quantification based on a twocompartment Tofts model (70) analysis of ICG uptake, finding a promising relationship between decreased perfusion $24 \mathrm{~h}$ after a single dose of 10 Gy and early treatment response in xenograft models (63). Similar trends were reported when changing the breathing gas of the mouse from air to $100 \%$ oxygen while imaging and measuring the change in $\mathrm{sO}_{2}\left(\Delta \mathrm{sO}_{2}\right)(44,45)$, which decreased after treatment (63). PAI using ICG is highly applicable in a clinical setting, since ICG is a clinically approved agent and DCEPAI has already been shown to be feasible in humans to image finger vasculature (71), to image lymphatic vessels of the lower (72) and upper limbs (73) in 3D, and used to assess metastatic status of lymph nodes in melanoma with PAI in humans $(74,75)$.

In the current workflow for image-guided EBRT, cone-beam CT, anatomical MRI and ultrasound are all used to ensure correct



FIGURE 1 | Macroscopic and mesoscopic photoacoustic imaging can monitor treatment-induced vascular changes and disease stage. (A) Multispectral optoacoustic tomography (MSOT)-derived quantitative blood oxygen saturation map $\left(\mathrm{sO}_{2}\right)$ overlaid on co-registered ultrasound axial slice of a head \& neck patient-derived xenograft tumor before and after a single dose of 15 Gy (top panel), and before and after combined 7.5 Gy of radiotherapy and administration of chemotherapeutic cetuximab. Increased $\mathrm{sO}_{2} 24 \mathrm{~h}$ after treatment was associated with decreased tumor volume two weeks later. (B) Hemodynamic stimulation challenge of salivary glands before and after a single dose of 15 Gy with decreased change in $\mathrm{sO}_{2}$ response post-radiotherapy suggesting radiation-induced damage. (C) Clinical $\mathrm{XZ}$ maximal intensity projection of mesoscopic PAl of graded atopic dermatitis in human skin. Vascular and structural scoring could accurately grade dermatitis and such score could potentially be translated for grading radiation-induced toxicity in RT. Panels (A, B) adapted from Rich et al. (60), and panel (C) adapted from Yew et al. (61). EP, Epidermis; DR, Dermis; LF, low frequency; HF, high frequency; RT, radiotherapy; \% $\mathrm{sO}_{2}$, percent blood oxygen saturation; CRT, chemoradiotherapy. 
localization of the tumor target and critical organs at risk structures. Additional imaging techniques that provide physiological and biological information regarding tumor and normal tissue response, namely, PET and MRI, are more often combined with standard image guidance in the context of research studies. Considering translation to a clinical context, PAI systems can be fast, cheap and portable, which could enable in-room imaging at the bedside compared to these other functional or molecular imaging modalities (Figure 2A). By scaling the resolution linearly with penetration depth (76), PAI offers a flexible approach to imaging vascular features in vivo (Figure 2B). PAI systems are capable of capturing information on $\mathrm{Hb}$ and $\mathrm{HbO}_{2}$ content at sub-100 $\mu \mathrm{m}$ resolution in individual blood vessels at superficial $(\sim 1 \mathrm{~mm})$ depths (77), or in whole tumors at few $\mathrm{cm}$ depths $(45,50)$. PAI is also readily combined with ultrasound (Figure 2C), which provides intrinsically co-registered anatomical information. The spatial resolution scale achievable with PAI provides a distinct understanding of vascular features in the tumor microenvironment locally, compared to PET for instance. While the length scale of quantitative PET has shown potential for voxel-level dose painting in precision radiotherapy, the finer length scale of PAI both at the macroscopic and mesoscopic scales could potentially provide a mechanistic understanding of tumor vasculature response to EBRT. Moreover, PAI measurements at multiple timepoints are much more feasible during a course of fractionated EBRT than PET or MRI, as PAI can be performed using a portable device in the radiotherapy department and even on-set in the treatment position. PAI systems could be readily deployed between fractions to detect changes in $\mathrm{sO}_{2}$ that could indicate response, thus providing initial radiation response assessment in-room during radiation fraction delivery. PAI measurements taken in real-time at bedside could inform on oxygen depletion through induction of DNA double strand breaks and the presence of hypoxia, indicating a need for dose modification (63). Although traditional fractionation of $~ 2 \mathrm{~Gy}$ daily, routinely used for these tumors, does not lead to the dramatic vascular response caused by ablative regimes (55), the overall length of the treatment is expected to generate measurable changes, and also providing an opportunity for mid-course adaptation. Nevertheless, further preclinical studies are needed to thoroughly examine modulation of PAI biomarkers in response to both conventional and hypofractionated regimes, in order to better estimate its clinical applications and potentials in the different steps of the radiotherapy framework (Figure 2D).

\section{Targeting Intratumoral Hypoxia With Photoacoustic Imaging Guidance}

Intratumoral heterogeneity complicates treatments in radiation oncology. The presence of focal hypoxia is clinically associated with cellular heterogeneity, genomic instability $(78,79)$, poor prognosis (80) and importantly, poor response to EBRT, particularly in cancers of hormone-sensitive tissues such as the breast $(81,82)$. Cells within these regions of focal hypoxia will activate hypoxia-inducible factors, which drive the transcription of multiple genes involved in cell growth, metabolism and angiogenesis $(48,83)$. One such factor is the vascular endothelial growth factor (VEGF), which plays a central role in stimulating endothelial cells to proliferate, sprout and form new blood vessels (84). Overexpression of VEGF often leads to an imbalance of pro- and anti-angiogenic factors, which results in a chaotic and heterogeneous network of blood vessels, namely, many immature vessels with poor pericyte coverage, irregular branching and a tortuous morphology $(85,86)$. Tumor vascular networks are consequently often poorly perfused, which can



FIGURE 2 | The potential role of photoacoustic imaging in the clinical radiotherapy framework. (A) Portable bedside PAl can be employed in-room before and/or after RT fractions due to its accessibility, portability and fast acquisitions. (B) PAl could map vascular features of tumors across scales, including blood oxygen saturation. (C) Dual ultrasound and PAl systems provide combined anatomical and molecular imaging features. (D) PAl could be introduced in the clinical RT framework pre-treatment, for diagnostics and pre-operative patient stratification, or for predictive imaging in parallel with CT simulation for radiation dose modulation. During radiotherapy, PAl could be used for monitoring response in the treatment room. After radiotherapy, PAl could further monitor tumor response based on blood oxygen saturation evaluations, which have been associated with local tumor control. PAl could also provide information for response assessment and insights into radiation-induced toxicity at early timepoints. Panel (C) provided in kind by Dr. Oshaani Abeyakoon. Created with BioRender. 
generate additional acute and transient hypoxia dynamics further associated with radioresistance (87).

Local tumor control with EBRT can be improved in patients with well-oxygenated tumors (88). The ability to differentiate normoxic from hypoxic tumor regions could thus improve prediction of EBRT outcomes (89), while appropriate image-guided radiation dose modulation could enhance EBRT cancer cell kill by enabling dose escalation to hypoxic regions (90). Unfortunately, while tumor hypoxia has been known as a key limiting factor in the efficacy of radiation for decades, it has yet to be incorporated in the clinical pipeline (91) due to challenges with availability and complexity of the current clinical approaches to hypoxia imaging.

Imaging the aberrant tumor vasculature with PAI has been proposed as a surrogate means to interrogate tumor hypoxia. Preclinical studies in mouse models of cancer have demonstrated that tomographic PAI measurements of $\mathrm{THb}$ and $\mathrm{sO}_{2}$ can inform on the heterogeneity of tumor hypoxia at $\sim 200 \mu \mathrm{m}$ resolution (45, 47, 48, 50, 92-97). PAI reveals lower $\mathrm{sO}_{2}$ in tumors compared to normal tissue, which is due to the imbalance of blood oxygen supply and tissue oxygen consumption in tumors (94). In multiple cancer mouse models, PAI estimation of $\mathrm{sO}_{2}$ correlated negatively with tumor hypoxia, validated ex vivo (50, 94-96). The PAI biomarker $\Delta \mathrm{sO}_{2}$ assessed under gas challenge enables a further robust assessment of the complex dynamics of tumor vessel perfusion, permeability and vasoactivity $(44,45)$. Importantly, low $\mathrm{sO}_{2}$ and $\Delta \mathrm{sO}_{2}$ spatially correlate with regions of tissue hypoxia and necrosis $(44,95)$. Taken together, these studies suggest that PAI maps may reveal intratumoral hypoxia at sufficient resolution to guide dose escalation or de-escalation assessments in a targeted therapy approach.

Key targets for potential deployment of PAI in the context of EBRT would be in head and neck (H\&N) and breast cancers. Ultrasound is already recommended in H\&N cancer to detect and delineate thyroid masses or tumors arising in the neck, and to identify local adenopathy in lymph nodes of the neck (98) before EBRT may be prescribed. In recent meta-analyses, ultrasound has also been recommended in breast cancer for palpable mass detection, especially in low-resource settings (99), and also in addition to mammography, providing increased sensitivity (100). Moreover, clinical PAI mammoscopy has been investigated in a diagnostic context (101) to: enable patient stratification based on intratumoral vascular features characterization (102-104); distinguish molecular subtypes (105); and for diagnostics in patients with dense breasts $(103,106)$, providing improved lesion detection when combined with integrated ultrasound system (107). Dual ultrasound and PAI systems have also demonstrated higher $\mathrm{THb}$ in patient breast tumors compared to normal tissue (108) and enabled visualization of vessels radiating from the tumor mass (109).

Since both H\&N and breast cancers are widely treated with EBRT, PAI-based assessment of radiation response is already being investigated in registered and recruiting clinical trials on $\mathrm{H} \& \mathrm{~N}$ cancer (ClinicalTrials.gov ID NCT04428515, NCT04110249, and NCT04437030). Nonetheless, the introduction of PAI to escalate dose to hypoxic regions in EBRT requires image co-registration between handheld PAI and planning CT, and would also benefit from co-registration of PAI between fractions to observe local differences in oxygenation. Ultrasound has been investigated for inter-fraction motion management in radiotherapy $(110,111)$ and fusion to CT for different applications $(112,113)$. Some promising studies have identified ways of matching skin surface and organ edges on both ultrasound and CT contrasts to co-register images with good similarity in the context of radiotherapy simulation (114, $115)$ and with probe tracking for intrafraction guidance $(116,117)$, with an extensive review reported elsewhere (118). Accurate mapping of regions on PAI to planning $\mathrm{CT}$ requires the registration of $\mathrm{PAI} /$ ultrasound system to the room coordinates using probe localization in the CT simulation suite or in the treatment room $(116,118,119)$. Nevertheless, promising findings in the context of ultrasound suggest that this challenge is not insurmountable. Overall, intra- and interfraction PAI monitoring of the tumor microenvironment opens new avenues for live assessment of tumor response to EBRT and dose adjustment based on local differences in intratumoral oxygenation, potentially increasing treatment control, especially in hypoxic tumors.

\section{Radiation-Induced Toxicity Assessment With Multi-Scale Photoacoustic Imaging}

Healthy skin toxicity is a common side effect of radiation, namely, acute dermatitis, burns and inflammation (120), and also chronic changes that may be permanent. Their rapid diagnosis and characterization are crucial for effective control of adverse radiation effects. Unfortunately, the first clinical signs may take weeks or months to appear (11). As skin damage appears during the course of fractionated EBRT, some early signs in the skin vasculature could be detected prior to the appearance of erythema ( 2 weeks) as damaged cells migrate to skin surface, or dry desquamation (i.e., skin peeling, $\sim 4$ weeks).

At present, clinical management of radiation-induced skin side effects with the Radiation Therapy Oncology Group (RTOG) scoring criteria is limited to the subjective visual assessment of visible clinical signs over weeks (11). For early detection of adverse effects of radiation to healthy skin, changes in $\mathrm{HbO}_{2}$ distribution have been shown to precede clinical symptoms, detectable with cutaneous blood flow measurements (121) and characterized in vivo with optical imaging modalities, namely, two-photon microscopy (122), diffuse optical tomography (123), and diffuse reflectance spectroscopy $(124,125)$. Similarly, B-mode ultrasound has already been studied in the context of radiation-induced toxicity, showing predictive parameters consistent with RTOG scores clinically (126).

The addition of PAI contrast to ultrasound parameters for direct measurements of vascularization and blood oxygenation using dual PAI/ultrasound systems or superficial PAI alone, may further assist evaluation of the early signs of acute radiationinduced toxicity and allow for their effective treatment. For example, in a salivary gland stimulation challenge conducted in mouse models, a decrease in $\mathrm{sO}_{2}$ change between measurements taken before and after salivary stimulation post-EBRT was associated with radiation-induced salivary gland toxicity in a murine model assessed with macroscopic PAI (Figure 1B) (60). Mesoscopic implementations of PAI can achieve $20 \mu \mathrm{m}$ inplane resolution up to $\sim 3 \mathrm{~mm}$ in depth for skin imaging (52, 
127), indicating potential for clinical skin toxicity assessment (128). Preliminary work on skin atopic dermatitis grading (61) showed that combining PAI mesoscopy-derived total blood volume, average vessel diameter, and ratio of low to high frequency signals gave a discriminating signature for atopic skin dermatitis grade (Figure 1C) (129). Beyond vascular imaging, the emerging capabilities of PAI for fibrosis imaging (129-131) may aid characterization of this late-stage skin toxicity manifestation $(12,120)$. In addition, PAI has shown promise for assessment of burns (132), wound healing (133, 134), and skin disorders such as psoriasis (135), all of which present with features similar to those in radiation-induced injuries. These studies highlight the promising potential of PAI to evaluate and assess vascular changes caused by radiation.

Taken together, the existing proven capabilities of clinical PAI for the characterization of microvascular abnormalities and inflammatory reactions (136), suggests the potential for clinical application of the technology to assessment of radiation skin toxicity.

\section{OUTLOOK}

Preclinical studies have already indicated the promise of multi-scale PAI in radiation oncology, which motivates further research in both the preclinical and clinical settings. Accounting for the limited penetration depth of PAI, while ensuring clinical relevance, will require targeting accessible sites such as $\mathrm{H} \& \mathrm{~N}$, breast or skin lesions and associated superficial lymph node masses. In the preclinical setting, validation of PAI biomarkers in clinically relevant EBRT schedules for specific human cancer models is needed. Since the timings of intratumoral oxygenation modulation during EBRT fractionation show distinct profiles for different tumor models and fractionation schemes, longitudinal PAI assessment of response is needed to further guide clinical study designs and to assess the potential of both OE- and DCE-PAI biomarkers longitudinally.

In the clinical setting, to introduce PAI in the clinical simulation process of the radiotherapy workflow, end-to-end frameworks for in-room probe tracking with optimal co-registration software of PAI to planning CT need to be developed and validated. Furthermore, extensive assessment of biomarker reproducibility and repeatability will be needed before deployment for radiotherapy dose planning. Since photoacoustic signals are highly dependent on tissue properties in the light path of the imaged region of interest, accurate characterization of tissue absorption and light fluence effects at depth must be conducted if quantitative imaging biomarkers are to be derived. For instance, the impact of skin tone, or melanin concentration, on $\mathrm{sO}_{2}$ measurements needs to be assessed, since it can lead to image artefacts and incorrect estimations of chromophore concentrations in deeper tissues. Spectral coloring induced by the characteristic absorption of melanin in skin layers has been shown to impact the quantification of PAI biomarkers at depth in tissue in silico and in phantoms (137). Interestingly, a significant difference was reported in $\mathrm{sO}_{2}$ measured in silico for the same imaged object between the lightest and darkest tested pigmentation at the surface (137), with the same order of magnitude of the difference in arterial oxygenation saturation reported between white and black patients in a recent report assessing pulse oximetry biases to skin pigmentation (138). In a single wavelength system, the increased melanin concentration in the forearms of living subjects was associated with a decrease of PAI signal at depth and in a significantly different characterization of vascular structures in the skin (139). Understanding these effects in vivo is important and could enable a quantitative framework for data correction to be realized before PAI biomarkers become widely employed clinically.

Similarly, measurements taken with hand-held imaging modalities, such as ultrasound, are known to be operatordependent. Both volume displacement and blood flow changes can be observed based on operator pressure in Doppler ultrasound and for different applications $(140,141)$. Such variability could be minimized in PAI through procedure standardization or by using non-handheld systems such as photoacoustic mammoscopes for breast imaging $(101,142)$, or a fixed probe on a mount with inroom infrared camera tracking, similar to previously developed and commercialized intrafraction ultrasound guidance systems (117). Variability induced by physiological processes such as breathing can also be controlled through breath-hold techniques or by tracking respiratory motion with optical surface guidance for instance (143), and the impact on imaging can be accounted for through intra-PAI co-registration with tomographic breathing detection (144). Trained radiographers and radiation therapists would have a key role in conducting these measurements and appropriate training would be essential in ensuring reproducibility. Preliminary assessment of PAI repeatability and reproducibility has been undertaken, suggesting good stability of repeated macroscopic PAI measurements in vivo (145). Future developments and cooperation between national and international bodies such as the International Photoacoustic Standardization Consortium (IPASC) $(146,147)$, the Quantitative Imaging Network (QIN) from the National Cancer Institute (148), and the Quantitative Imaging Biomarkers Alliance (QIBA) from the Radiological Society of North America (149) will be essential on the path of clinical translation (150).

Overall, PAI shows potential for providing predictive response biomarkers pre-EBRT and enabling assessment of vascular changes both in the tumor and in healthy irradiated skin after radiation exposure, highly relevant for detecting treatment response and modulating fractionated therapy. Furthermore, thanks to the non-ionizing nature and portability of PAI, these examinations could be repeated throughout treatment at bedside to enable longitudinal assessment of hypoxia during EBRT, especially for hypofractionated regimens such as SBRT and SABR. Integrating multi-scale PAI in radiation oncology with existing imaging modalities, from treatment guidance to early tumor response assessment and radiation toxicity, could therefore open new paradigms in the future of radiation oncology.

\section{DATA AVAILABILITY STATEMENT}

The original contributions presented in the study are included in the article/supplementary material. Further inquiries can be directed to the corresponding author. 


\section{AUTHOR CONTRIBUTIONS}

All authors listed have made a substantial, direct, and intellectual contribution to the work and approved it for publication.

\section{FUNDING}

All authors were supported by the Cancer Research UK under grant numbers C14303/A17197, C9545/A29580, C47594/ A16267, C197/A16465, C47594/A29448 and in particular by the Cancer Research UK RadNet Cambridge under grant

\section{REFERENCES}

1. Chandra RA, Keane FK, Voncken FEM, Thomas CRJr. Contemporary Radiotherapy: Present and Future. Lancet (2021) 398:171-84. doi: 10.1016/S0140-6736(21)00233-6

2. Corradini S, Krug D, Meattini I, Matuschek C, Bolke E, Francolini G, et al. Preoperative Radiotherapy: A Paradigm Shift in the Treatment of Breast Cancer? A Review of Literature. Crit Rev Oncol Hematol (2019) 141:102-11. doi: 10.1016/j.critrevonc.2019.06.003

3. Speers C, Pierce LJ. Postoperative Radiotherapy After Breast-Conserving Surgery for Early-Stage Breast Cancer: A Review. JAMA Oncol (2016) 2:1075-82. doi: 10.1001/jamaoncol.2015.5805

4. Alterio D, Marvaso G, Ferrari A, Volpe S, Orecchia R, Jereczek-Fossa BA. Modern Radiotherapy for Head and Neck Cancer. Semin Oncol (2019) 46:233-45. doi: 10.1053/j.seminoncol.2019.07.002

5. Castaneda SA, Strasser J. Updates in the Treatment of Breast Cancer With Radiotherapy. Surg Oncol Clin N Am (2017) 26:371-82. doi: 10.1016/ j.soc.2017.01.013

6. Eisenhauer EA, Therasse P, Bogaerts J, Schwartz LH, Sargent D, Ford R, et al. New Response Evaluation Criteria in Solid Tumours: Revised RECIST Guideline (Version 1.1). Eur J Cancer (2009) 45:228-47. doi: 10.1016/ j.ejca.2008.10.026

7. Seymour L, Bogaerts J, Perrone A, Ford R, Schwartz LH, Mandrekar S, et al. iRECIST: Guidelines for Response Criteria for Use in Trials Testing Immunotherapeutics. Lancet Oncol (2017) 18:e143-52. doi: 10.1016/ S1470-2045(17)30074-8

8. Tirkes T, Hollar MA, Tann M, Kohli MD, Akisik F, Sandrasegaran K. Response Criteria in Oncologic Imaging: Review of Traditional and New Criteria. Radiographics (2013) 33:1323-41. doi: 10.1148/rg.335125214

9. Kurra V, Sullivan RJ, Gainor JF, Hodi FS, Gandhi L, Sadow CA, et al. Pseudoprogression in Cancer Immunotherapy: Rates, Time Course and Patient Outcomes. J Clin Oncol (2016) 34:6580-0. doi: 10.1200/ JCO.2016.34.15_suppl.6580

10. Gerwing M, Herrmann K, Helfen A, Schliemann C, Berdel WE, Eisenblatter $\mathrm{M}$, et al. The Beginning of the End for Conventional RECIST - Novel Therapies Require Novel Imaging Approaches. Nat Rev Clin Oncol (2019) 16:442-58. doi: 10.1038/s41571-019-0169-5

11. Naylor W, Mallett J. Management of Acute Radiotherapy Induced Skin Reactions: A Literature Review. Eur J Oncol Nurs (2001) 5:221-33. doi: 10.1054/ejon.2001.0145

12. Straub JM, New J, Hamilton CD, Lominska C, Shnayder Y, Thomas SM. Radiation-Induced Fibrosis: Mechanisms and Implications for Therapy. $J$ Cancer Res Clin Oncol (2015) 141:1985-94. doi: 10.1007/s00432-015-1974-6

13. Wahl RL, Jacene H, Kasamon Y, Lodge MA. From RECIST to PERCIST: Evolving Considerations for PET Response Criteria in Solid Tumors. J Nucl Med (2009) 50 Suppl:1, 122S-150S. doi: 10.2967/jnumed.108.057307

14. Caldwell CB, Mah K, Ung YC, Danjoux CE, Balogh JM, Ganguli SN, et al. Observer Variation in Contouring Gross Tumor Volume in Patients With Poorly Defined Non-Small-Cell Lung Tumors on CT: The Impact of 18FDG-Hybrid PET Fusion. Int J Radiat Oncol Biol Phys (2001) 51:92331. doi: 10.1016/S0360-3016(01)01722-9 number C17918/A28870. TL is supported by the Cambridge Trust. LH is funded from NPL's MedAccel programme financed by the Department of Business, Energy and Industrial Strategy's Industrial Strategy Challenge Fund.

\section{ACKNOWLEDGMENTS}

The authors would like to acknowledge the contribution of Oshaani Abeyakoon for acquiring clinical optoacoustic data shown in Figure 2, and of Sabrina Terranova for the insightful discussions and for kindly reviewing our manuscript.

15. Nimmagadda S, Ford EC, Wong JW, Pomper MG. Targeted Molecular Imaging in Oncology: Focus on Radiation Therapy. Semin Radiat Oncol (2008) 18:136-48. doi: 10.1016/j.semradonc.2007.10.009

16. Toma-Dasu I, Uhrdin J, Antonovic L, Dasu A, Nuyts S, Dirix P, et al. Dose Prescription and Treatment Planning Based on FMISO-PET Hypoxia. Acta Oncol (2012) 51:222-30. doi: 10.3109/0284186X.2011.599815

17. Lee NY, Mechalakos JG, Nehmeh S, Lin Z, Squire OD, Cai S, et al. Fluorine18-Labeled Fluoromisonidazole Positron Emission and Computed Tomography-Guided Intensity-Modulated Radiotherapy for Head and Neck Cancer: A Feasibility Study. Int J Radiat Oncol Biol Phys (2008) 70:2-13. doi: 10.1016/j.ijrobp.2007.06.039

18. Lee N, Schoder H, Beattie B, Lanning R, Riaz N, McBride S, et al. Strategy of Using Intratreatment Hypoxia Imaging to Selectively and Safely Guide Radiation Dose De-Escalation Concurrent With Chemotherapy for Locoregionally Advanced Human Papillomavirus-Related Oropharyngeal Carcinoma. Int J Radiat Oncol Biol Phys (2016) 96:9-17. doi: 10.1016/ j.ijrobp.2016.04.027

19. Horsman MR, Mortensen LS, Petersen JB, Busk M, Overgaard J. Imaging Hypoxia to Improve Radiotherapy Outcome. Nat Rev Clin Oncol (2012) 9:674-87. doi: 10.1038/nrclinonc.2012.171

20. Michiels C, Tellier C, Feron O. Cycling Hypoxia: A Key Feature of the Tumor Microenvironment. Biochim Biophys Acta (2016) 1866:76-86. doi: 10.1016/j.bbcan.2016.06.004

21. Grimes DR. M. Partridge, A Mechanistic Investigation of the Oxygen Fixation Hypothesis and Oxygen Enhancement Ratio. BioMed Phys Eng Express (2015) 1:045209. doi: 10.1088/2057-1976/1/4/045209

22. Carlson DJ, Keall PJ, Loo BWJr., Chen ZJ, Brown JM. Hypofractionation Results in Reduced Tumor Cell Kill Compared to Conventional Fractionation for Tumors With Regions of Hypoxia. Int J Radiat Oncol Biol Phys (2011) 79:1188-95. doi: 10.1016/j.ijrobp.2010.10.007

23. Brown JM, Carlson DJ, Brenner DJ. The Tumor Radiobiology of SRS and SBRT: Are More Than the 5 Rs Involved? Int J Radiat Oncol Biol Phys (2014) 88:254-62. doi: 10.1016/j.ijrobp.2013.07.022

24. Brown JM, Diehn M, Loo BWJr. Stereotactic Ablative Radiotherapy Should be Combined With a Hypoxic Cell Radiosensitizer. Int J Radiat Oncol Biol Phys (2010) 78:323-7. doi: 10.1016/j.ijrobp.2010.04.070

25. Span PN, Bussink J. Biology of Hypoxia. Semin Nucl Med (2015) 45:101-9. doi: 10.1053/j.semnuclmed.2014.10.002

26. D'Alonzo RA, Gill S, Rowshanfarzad P, Keam S, MacKinnon KM, Cook AM, et al. In Vivo Noninvasive Preclinical Tumor Hypoxia Imaging Methods: A Review. Int I Radiat Biol (2021) 97:593-631. doi: 10.1080/09553002.2021.1900943

27. Wiedenmann N, Grosu AL, Buchert M, Rischke HC, Ruf J, Bielak L, et al. The Utility of Multiparametric MRI to Characterize Hypoxic Tumor Subvolumes in Comparison to FMISO PET/CT. Consequences for Diagnosis and Chemoradiation Treatment Planning in Head and Neck Cancer. Radiother Oncol (2020) 150:128-35. doi: 10.1016/ j.radonc.2020.06.013

28. Hompland T, Hole KH, Ragnum HB, Aarnes EK, Vlatkovic L, Lie AK, et al. Combined MR Imaging of Oxygen Consumption and Supply Reveals Tumor Hypoxia and Aggressiveness in Prostate Cancer Patients. Cancer Res (2018) 78:4774-85. doi: 10.1158/0008-5472.CAN-17-3806 
29. Hillestad T, Hompland T, Fjeldbo CS, Skingen VE, Salberg UB, Aarnes EK, et al. MRI Distinguishes Tumor Hypoxia Levels of Different Prognostic and Biological Significance in Cervical Cancer. Cancer Res (2020) 80:3993-4003. doi: 10.1158/0008-5472.CAN-20-0950

30. Li A, Andersen E, Lervåg C, Julin CH, Lyng H, Hellebust TP, et al. Dynamic Contrast Enhanced Magnetic Resonance Imaging for Hypoxia Mapping and Potential for Brachytherapy Targeting. Phys Imaging Radiat Oncol (2017) 2:1-6. doi: 10.1016/j.phro.2017.03.002

31. Her EJ, Haworth A, Sun Y, Williams S, Reynolds HM, Kennedy A, et al. Biologically Targeted Radiation Therapy: Incorporating Patient-Specific Hypoxia Data Derived From Quantitative Magnetic Resonance Imaging. Cancers (Basel) (2021) 13(19):4897-912. doi: 10.3390/cancers13194897

32. Arai TJ, Yang DM, Campbell JW, Chiu T, Cheng X, Stojadinovic S, et al. Oxygen-Sensitive MRI: A Predictive Imaging Biomarker for Tumor Radiation Response? Int J Radiat Oncol Biol Phys (2021) 110:1519-29. doi: 10.1016/j.ijrobp.2021.03.039

33. Rodrigues LM, Howe FA, Griffiths JR, Robinson SP. Tumor R2* Is a Prognostic Indicator of Acute Radiotherapeutic Response in Rodent Tumors. J Magnetic Resonance Imaging (2004) 19:482-8. doi: 10.1002/ jmri.20024

34. Li M, Zhang Q, Yang K. Role of MRI-Based Functional Imaging in Improving the Therapeutic Index of Radiotherapy in Cancer Treatment. Front Oncol (2021) 11:645177. doi: 10.3389/fonc.2021.645177

35. Heiken JP. Contrast Safety in the Cancer Patient: Preventing ContrastInduced Nephropathy. Cancer Imaging (2008) 8 Spec No A:S124-127. doi: 10.1102/1470-7330.2008.9018

36. Rogosnitzky M, Branch S. Gadolinium-Based Contrast Agent Toxicity: A Review of Known and Proposed Mechanisms. Biometals (2016) 29:365-76. doi: 10.1007/s10534-016-9931-7

37. Chin S, Eccles CL, McWilliam A, Chuter R, Walker E, Whitehurst P, et al. Magnetic Resonance-Guided Radiation Therapy: A Review. J Med Imaging Radiat Oncol (2020) 64:163-77. doi: 10.1111/1754-9485.12968

38. Thorwarth D, Low DA. Technical Challenges of Real-Time Adaptive MRGuided Radiotherapy. Front Oncol (2021) 11:634507. doi: 10.3389/ fonc.2021.634507

39. Shirvani SM, Huntzinger CJ, Melcher T, Olcott PD, Voronenko Y, BartlettRoberto J, et al. Biology-Guided Radiotherapy: Redefining the Role of Radiotherapy in Metastatic Cancer. Br J Radiol (2021) 94:20200873. doi: $10.1259 /$ bjr.20200873

40. Oderinde OM, Shirvani SM, Olcott PD, Kuduvalli G, Mazin S, Larkin D. The Technical Design and Concept of a PET/CT Linac for Biology-Guided Radiotherapy. Clin Transl Radiat Oncol (2021) 29:106-12. doi: 10.1016/ j.ctro.2021.04.003

41. Geethanath S, Vaughan JTJr. Accessible Magnetic Resonance Imaging: A Review. J Magn Reson Imaging (2019) 49:e65-77. doi: 10.1002/jmri.26638

42. Wang LV, Yao J. A Practical Guide to Photoacoustic Tomography in the Life Sciences. Nat Methods (2016) 13:627-38. doi: 10.1038/nmeth.3925

43. Ntziachristos V, Pleitez MA, Aime S, Brindle KM. Emerging Technologies to Image Tissue Metabolism. Cell Metab (2019) 29:518-38. doi: 10.1016/ j.cmet.2018.09.004

44. Tomaszewski MR, Gehrung M, Joseph J, Quiros-Gonzalez I, Disselhorst JA, Bohndiek SE. Oxygen-Enhanced and Dynamic Contrast-Enhanced Optoacoustic Tomography Provide Surrogate Biomarkers of Tumor Vascular Function, Hypoxia, and Necrosis. Cancer Res (2018) 78:5980-91. doi: 10.1158/0008-5472.CAN-18-1033

45. Tomaszewski MR, Gonzalez IQ, O'Connor JP, Abeyakoon O, Parker GJ, Williams KJ, et al. Oxygen Enhanced Optoacoustic Tomography (OE-OT) Reveals Vascular Dynamics in Murine Models of Prostate Cancer. Theranostics (2017) 7:2900-13. doi: 10.7150/thno.19841

46. Heijblom M, Piras D, van den Engh FM, van der Schaaf M, Klaase JM, Steenbergen W, et al. The State of the Art in Breast Imaging Using the Twente Photoacoustic Mammoscope: Results From 31 Measurements on Malignancies. Eur Radiol (2016) 26:3874-87. doi: 10.1007/s00330-016-4240-7

47. Bendinger AL, Glowa C, Peter J, Karger CP. Photoacoustic Imaging to Assess Pixel-Based So2 Distributions in Experimental Prostate Tumors. J BioMed Opt (2018) 23:1-11. doi: 10.1117/1.JBO.23.3.036009
48. Brown E, Brunker J, Bohndiek SE. Photoacoustic Imaging as a Tool to Probe the Tumour Microenvironment. Dis Model Mech (2019) 12(7):dmm039636. doi: $10.1242 / \mathrm{dmm} .039636$

49. Niederhauser JJ, Jaeger M, Lemor R, Weber P, Frenz M. Combined Ultrasound and Optoacoustic System for Real-Time High-Contrast Vascular Imaging In Vivo. IEEE Trans Med Imaging (2005) 24:436-40. doi: 10.1109/TMI.2004.843199

50. Quiros-Gonzalez I, Tomaszewski MR, Aitken SJ, Ansel-Bollepalli L, McDuffus LA, Gill M, et al. Optoacoustics Delineates Murine Breast Cancer Models Displaying Angiogenesis and Vascular Mimicry. $\mathrm{Br} J$ Cancer (2018) 118:1098-106. doi: 10.1038/s41416-018-0033-x

51. Knox HJ, Kim TW, Zhu Z, Chan J. Photophysical Tuning of N-Oxide-Based Probes Enables Ratiometric Photoacoustic Imaging of Tumor Hypoxia. ACS Chem Biol (2018) 13:1838-43. doi: 10.1021/acschembio.8b00099

52. Li D, Humayun L, Vienneau E, Vu T, Yao J. Seeing Through the Skin: Photoacoustic Tomography of Skin Vasculature and Beyond. JID Innovations (2021) 1:100039. doi: 10.1016/j.xjidi.2021.100039

53. Yao J, Maslov KI, Shi Y, Taber LA, Wang LV. In Vivo Photoacoustic Imaging of Transverse Blood Flow by Using Doppler Broadening of Bandwidth. Opt Lett (2010) 35:1419-21. doi: 10.1364/OL.35.001419

54. Eriksson D, Stigbrand T. Radiation-Induced Cell Death Mechanisms. Tumour Biol (2010) 31:363-72. doi: 10.1007/s13277-010-0042-8

55. Fuks Z, Kolesnick R. Engaging the Vascular Component of the Tumor Response. Cancer Cell (2005) 8:89-91. doi: 10.1016/j.ccr.2005.07.014

56. Singleton DC, Macann A, Wilson WR. Therapeutic Targeting of the Hypoxic Tumour Microenvironment. Nat Rev Clin Oncol (2021) 18 (12):751-72. doi: 10.1038/s41571-021-00539-4

57. Park HJ, Griffin RJ, Hui S, Levitt SH, Song CW. Radiation-Induced Vascular Damage in Tumors: Implications of Vascular Damage in Ablative Hypofractionated Radiotherapy (SBRT and SRS). Radiat Res (2012) 177:311-27. doi: 10.1667/RR2773.1

58. Brown SL, Nagaraja TN, Aryal MP, Panda S, Cabral G, Keenan KA, et al. MRI-Tracked Tumor Vascular Changes in the Hours After Single-Fraction Irradiation. Radiat Res (2015) 183:713-21. doi: 10.1667/RR13458.1

59. Zhou H, Zhang Z, Denney R, Williams JS, Gerberich J, Stojadinovic S, et al. Tumor Physiological Changes During Hypofractionated Stereotactic Body Radiation Therapy Assessed Using Multi-Parametric Magnetic Resonance Imaging. Oncotarget (2017) 8:37464-77. doi: 10.18632/oncotarget.16395

60. Rich LJ, Seshadri M. Photoacoustic Monitoring of Tumor and Normal Tissue Response to Radiation. Sci Rep (2016) 6:21237. doi: 10.1038/ srep21237

61. Yew YW, Unnimadhava Kurup Soudamini Amma D, Kuan AHY, Li X, Dev K, Ebrahim Attia AB, et al. Raster-Scanning Optoacoustic Mesoscopy Imaging as an Objective Disease Severity Tool in Atopic Dermatitis Patients. J Am Acad Dermatol (2021) 84:1121-3. doi: 10.1016/j.jaad.2020.06.045

62. Martinho Costa M, Shah A, Rivens I, Box C, O'Shea T, Bamber J, et al. Photoacoustic Imaging for the Prediction and Assessment of Response to Radiotherapy In Vivo. bioRxiv (2018). 1-30. doi: 10.1101/329516

63. Goel S, de la Cerda J, Schuler W, Kotrotsou A, Cárdenas-Rodríguez J, Pagel MD. Improving Evaluations of Radiation Therapy With Dynamic Contrast Enhanced Multispectral Optoacoustic Tomography (DCE MSOT). Proc Eur Mol Imaging Meeting PS 09-03 (2020).

64. Rich LJ, Miller A, Singh AK, Seshadri M. Photoacoustic Imaging as an Early Biomarker of Radio Therapeutic Efficacy in Head and Neck Cancer. Theranostics (2018) 8:2064-78. doi: 10.7150/thno.21708

65. Sun JM, Stapleton AS, Chen Y, Heinmiller A, Bate D, McGrath T, et al. Monitoring Radiation Response in Tumor Vasculature Using Intravital Photoacoustic Imaging in a Murine Window Chamber Model In Vivo. Proc 103rd Annu Meeting Am Assoc Cancer Res (2012) 72 (8_Supplement):4338. doi: 10.1158/1538-7445.AM2012-4338

66. Bussink J, Kaanders JHAM, Rijken PFJW, Raleigh JA, van der Kogel AJ. Changes in Blood Perfusion and Hypoxia After Irradiation of a Human Squamous Cell Carcinoma Xenograft Tumor Line. Radiat Res (2000) 153:398-404. doi: 10.1667/0033-7587(2000)153[0398:CIBPAH]2.0.CO;2

67. Hysi E, Fadhel MN, Wang Y, Sebastian JA, Giles A, Czarnota GJ, et al. Photoacoustic Imaging Biomarkers for Monitoring Biophysical Changes 
During Nanobubble-Mediated Radiation Treatment. Photoacoustics (2020) 20:100201. doi: 10.1016/j.pacs.2020.100201

68. Bohndiek SE, Sasportas LS, Machtaler S, Jokerst JV, Hori S, Gambhir SS. Photoacoustic Tomography Detects Early Vessel Regression and Normalization During Ovarian Tumor Response to the Antiangiogenic Therapy Trebananib. J Nucl Med (2015) 56:1942-7. doi: 10.2967/jnumed.115.160002

69. Hupple CW, Morscher S, Burton NC, Pagel MD, McNally LR, CardenasRodriguez J. A Light-Fluence-Independent Method for the Quantitative Analysis of Dynamic Contrast-Enhanced Multispectral Optoacoustic Tomography (DCE MSOT). Photoacoustics (2018) 10:54-64. doi: 10.1016/ j.pacs.2018.04.003

70. Tofts PS, Brix G, Buckley DL, Evelhoch JL, Henderson E, Knopp MV, et al. Estimating Kinetic Parameters From Dynamic Contrast-Enhanced T(1)Weighted MRI of a Diffusable Tracer: Standardized Quantities and Symbols. J Magn Reson Imaging (1999) 10:223-32. doi: 10.1002/(SICI)1522-2586 (199909) 10:3<223::AID-JMRI2>3.0.CO;2-S

71. Lutzweiler C, Meier R, Rummeny E, Ntziachristos V, Razansky D. RealTime Optoacoustic Tomography of Indocyanine Green Perfusion and Oxygenation Parameters in Human Finger Vasculature. Opt Lett (2014) 39:4061-4. doi: 10.1364/OL.39.004061

72. Suzuki Y, Kajita H, Konishi N, Oh A, Urano M, Watanabe S, et al. Subcutaneous Lymphatic Vessels in the Lower Extremities: Comparison Between Photoacoustic Lymphangiography and Near-Infrared Fluorescence Lymphangiography. Radiology (2020) 295:469-74. doi: 10.1148/ radiol.2020191710

73. Kajita H, Kishi K. High-Resolution Imaging of Lymphatic Vessels With Photoacoustic Lymphangiography. Radiology (2019) 292:35. doi: 10.1148/ radiol.2019190241

74. Stoffels I, Morscher S, Helfrich I, Hillen U, Leyh J, Burton NC, et al. Metastatic Status of Sentinel Lymph Nodes in Melanoma Determined Noninvasively With Multispectral Optoacoustic Imaging. Sci Transl Med (2015) 7:317ra199. doi: 10.1126/scitranslmed.aad1278

75. Stoffels I, Jansen P, Petri M, Goerdt L, Brinker TJ, Griewank KG, et al. Assessment of Nonradioactive Multispectral Optoacoustic Tomographic Imaging With Conventional Lymphoscintigraphic Imaging for Sentinel Lymph Node Biopsy in Melanoma. JAMA Netw Open (2019) 2:e199020e199020. doi: 10.1001/jamanetworkopen.2019.9020

76. Ntziachristos V. Going Deeper Than Microscopy: The Optical Imaging Frontier in Biology. Nat Methods (2010) 7:603-14. doi: 10.1038/nmeth.1483

77. Laufer J, Johnson P, Zhang E, Treeby B, Cox B, Pedley B, et al. In Vivo Preclinical Photoacoustic Imaging of Tumor Vasculature Development and Therapy. J BioMed Opt (2012) 17:056016. doi: 10.1117/1.JBO.17.5.056016

78. Lloyd MC, Cunningham JJ, Bui MM, Gillies RJ, Brown JS, Gatenby RA, et al. Darwinian Dynamics of Intratumoral Heterogeneity: Not Solely Random Mutations But Also Variable Environmental Selection Forces. Cancer Res (2016) 76:3136-44. doi: 10.1158/0008-5472.CAN-15-2962

79. Pires IM, Bencokova Z, Milani M, Folkes LK, Li JL, Stratford MR, et al. Effects of Acute Versus Chronic Hypoxia on DNA Damage Responses and Genomic Instability. Cancer Res (2010) 70:925-35. doi: 10.1158/00085472.CAN-09-2715

80. Gillies RJ, Brown JS, Anderson ARA, Gatenby RA. Eco-Evolutionary Causes and Consequences of Temporal Changes in Intratumoural Blood Flow. Nat Rev Cancer (2018) 18:576-85. doi: 10.1038/s41568-018-0030-7

81. Trastour C, Benizri E, Ettore F, Ramaioli A, Chamorey E, Pouyssegur J, et al. HIF-1alpha and CA IX Staining in Invasive Breast Carcinomas: Prognosis and Treatment Outcome. Int J Cancer (2007) 120:1451-8. doi: 10.1002/ ijc. 22436

82. Lundgren K, Holm C, Landberg G. Hypoxia and Breast Cancer: Prognostic and Therapeutic Implications. Cell Mol Life Sci (2007) 64:3233-47. doi: 10.1007/s00018-007-7390-6

83. LaGory EL, Giaccia AJ. The Ever-Expanding Role of HIF in Tumour and Stromal Biology. Nat Cell Biol (2016) 18:356-65. doi: 10.1038/ncb3330

84. Hoeben A, Landuyt B, Highley MS, Wildiers H, Van Oosterom AT, De Bruijn EA. Vascular Endothelial Growth Factor and Angiogenesis. Pharmacol Rev (2004) 56:549-80. doi: 10.1124/pr.56.4.3

85. Ribeiro AL, Okamoto OK. Combined Effects of Pericytes in the Tumor Microenvironment. Stem Cells Int (2015) 2015:868475. doi: 10.1155/2015/ 868475
86. Krishna Priya S, Nagare RP, Sneha VS, Sidhanth C, Bindhya S, Manasa P, et al. Tumour Angiogenesis-Origin of Blood Vessels. Int J Cancer (2016) 139:729-35. doi: 10.1002/ijc.30067

87. Bristow RG, Hill RP. Hypoxia, DNA Repair and Genetic Instability. Nat Rev Cancer (2008) 8:180-92. doi: 10.1038/nrc2344

88. Overgaard J. Hypoxic Modification of Radiotherapy in Squamous Cell Carcinoma of the Head and Neck-A Systematic Review and Meta-Analysis. Radiother Oncol (2011) 100:22-32. doi: 10.1016/j.radonc.2011.03.004

89. Colliez F, Gallez B, Jordan BF. Assessing Tumor Oxygenation for Predicting Outcome in Radiation Oncology: A Review of Studies Correlating Tumor Hypoxic Status and Outcome in the Preclinical and Clinical Settings. Front Oncol (2017) 7:10. doi: 10.3389/fonc.2017.00010

90. Lin A, Hahn SM. Hypoxia Imaging Markers and Applications for Radiation Treatment Planning. Semin Nucl Med (2012) 42:343-52. doi: 10.1053/ j.semnuclmed.2012.04.002

91. Thiruthaneeswaran N, Bibby BAS, Yang L, Hoskin PJ, Bristow RG, Choudhury A, et al. Lost in Application: Measuring Hypoxia for Radiotherapy Optimisation. Eur J Cancer (2021) 148:260-76. doi: 10.1016/ j.ejca.2021.01.039

92. Chekkoury A, Nunes A, Gateau J, Symvoulidis P, Feuchtinger A, Beziere N, et al. High-Resolution Multispectral Optoacoustic Tomography of the Vascularization and Constitutive Hypoxemia of Cancerous Tumors. Neoplasia (2016) 18:459-67. doi: 10.1016/j.neo.2016.06.004

93. Li M, Oh J, Xie X, Ku G, Wang W, Li C, et al. Simultaneous Molecular and Hypoxia Imaging of Brain Tumors In Vivo Using Spectroscopic Photoacoustic Tomography. Proc IEEE (2008) 96:481-9. doi: 10.1109/ JPROC.2007.913515

94. Tzoumas S, Nunes A, Olefir I, Stangl S, Symvoulidis P, Glasl S, et al. Eigenspectra Optoacoustic Tomography Achieves Quantitative Blood Oxygenation Imaging Deep in Tissues. Nat Commun (2016) 7:12121. doi: $10.1038 /$ ncomms 12121

95. Gerling M, Zhao Y, Nania S, Norberg KJ, Verbeke CS, Englert B, et al. RealTime Assessment of Tissue Hypoxia In Vivo With Combined Photoacoustics and High-Frequency Ultrasound. Theranostics (2014) 4:604-13. doi: 10.7150/thno.7996

96. Bar-Zion A, Yin M, Adam D, Foster FS. Functional Flow Patterns and Static Blood Pooling in Tumors Revealed by Combined Contrast-Enhanced Ultrasound and Photoacoustic Imaging. Cancer Res (2016) 76:4320-31. doi: 10.1158/0008-5472.CAN-16-0376

97. Rich LJ, Sexton S, Curtin L, Seshadri M. Spatiotemporal Optoacoustic Mapping of Tumor Hemodynamics in a Clinically Relevant Orthotopic Rabbit Model of Head and Neck Cancer. Trans Oncol (2017) 10:839-45. doi: 10.1016/j.tranon.2017.08.004

98. Lewis-Jones H, Colley S, Gibson D. Imaging in Head and Neck Cancer: United Kingdom National Multidisciplinary Guidelines. J Laryngol Otology (2016) 130:S28-31. doi: 10.1017/S0022215116000396

99. Sood R, Rositch AF, Shakoor D, Ambinder E, Pool K-L, Pollack E, et al. Ultrasound for Breast Cancer Detection Globally: A Systematic Review and Meta-Analysis. J Global Oncol (2019) 5:1-17. doi: 10.1200/jgo.19.00127

100. Rebolj M, Assi V, Brentnall A, Parmar D, Duffy SW. Addition of Ultrasound to Mammography in the Case of Dense Breast Tissue: Systematic Review and Meta-Analysis. Br J Cancer (2018) 118:1559-70. doi: 10.1038/s41416-0180080-3

101. Manohar S, Dantuma M. Current and Future Trends in Photoacoustic Breast Imaging. Photoacoustics (2019) 16:100134. doi: 10.1016/j.pacs.2019.04.004

102. Diot G, Metz S, Noske A, Liapis E, Schroeder B, Ovsepian SV, et al. Multispectral Optoacoustic Tomography (MSOT) of Human Breast Cancer. Clin Cancer Res (2017) 23:6912-22. doi: 10.1158/1078-0432.CCR16-3200

103. Lin L, Hu P, Shi J, Appleton CM, Maslov K, Li L, et al. Single-Breath-Hold Photoacoustic Computed Tomography of the Breast. Nat Commun (2018) 9:2352. doi: 10.1038/s41467-018-04576-z

104. Menezes GLG, Pijnappel RM, Meeuwis C, Bisschops R, Veltman J, Lavin PT, et al. Downgrading of Breast Masses Suspicious for Cancer by Using Optoacoustic Breast Imaging. Radiology (2018) 288:355-65. doi: 10.1148/ radiol.2018170500

105. Dogan BE, Menezes GLG, Butler RS, Neuschler EI, Aitchison R, Lavin PT, et al. Optoacoustic Imaging and Gray-Scale US Features of Breast Cancers: 
Correlation With Molecular Subtypes. Radiology (2019) 292:564-72. doi: 10.1148/radiol.2019182071

106. Dean-Ben XL, Fehm TF, Gostic M, Razansky D. Volumetric Hand-Held Optoacoustic Angiography as a Tool for Real-Time Screening of Dense Breast. J Biophotonics (2016) 9:253-9. doi: 10.1002/jbio.201500008

107. Asao Y, Hashizume Y, Suita T, Nagae KI, Fukutani K, Sudo Y, et al. Photoacoustic Mammography Capable of Simultaneously Acquiring Photoacoustic and Ultrasound Images. J BioMed Opt (2016) 21:116009. doi: 10.1117/1.JBO.21.11.116009

108. Becker A, Masthoff M, Claussen J, Ford SJ, Roll W, Burg M, et al. Multispectral Optoacoustic Tomography of the Human Breast: Characterisation of Healthy Tissue and Malignant Lesions Using a Hybrid Ultrasound-Optoacoustic Approach. Eur Radiol (2018) 28:602-9. doi: 10.1007/s00330-017-5002-x

109. Neuschler EI, Butler R, Young CA, Barke LD, Bertrand ML, Bohm-Velez M, et al. A Pivotal Study of Optoacoustic Imaging to Diagnose Benign and Malignant Breast Masses: A New Evaluation Tool for Radiologists. Radiology (2018) 287:398-412. doi: 10.1148/radiol.2017172228

110. Krengli M, Loi G, Pisani C, Beldi D, Apicella G, Amisano V, et al. ThreeDimensional Surface and Ultrasound Imaging for Daily IGRT of Prostate Cancer. Radiat Oncol (2016) 11:159. doi: 10.1186/s13014-016-0734-3

111. Fargier-Voiron M, Presles B, Pommier P, Munoz A, Rit S, Sarrut D, et al. Evaluation of a New Transperineal Ultrasound Probe for Inter-Fraction Image-Guidance for Definitive and Post-Operative Prostate Cancer Radiotherapy. Phys Med (2016) 32:499-505. doi: 10.1016/j.ejmp.2016.01.481

112. Beer L, Martin-Gonzalez P, Delgado-Ortet M, Reinius M, Rundo L, Woitek R, et al. Ultrasound-Guided Targeted Biopsies of CT-Based Radiomic Tumour Habitats: Technical Development and Initial Experience in Metastatic Ovarian Cancer. Eur Radiol (2021) 31:3765-72. doi: 10.1007/ s00330-020-07560-8

113. D’Onofrio $\mathrm{M}$, Beleù $\mathrm{A}$, Gaitini $\mathrm{D}$, Corréas J-M, Brady $\mathrm{A}$, Clevert $\mathrm{D}$, et al. Abdominal Applications of Ultrasound Fusion Imaging Technique: Liver, Kidney, and Pancreas. Insights Into Imaging (2019) 10:6. doi: 10.1186/ s13244-019-0692-z

114. Wein W, Röper B, Navab N. Automatic Registration and Fusion of Ultrasound With CT for Radiotherapy. In: JS Duncan, G Gerig, editors. Medical Image Computing and Computer-Assisted Intervention - MICCAI 2005. Berlin, Heidelberg: Springer Berlin Heidelberg (2005). p. 303-11.

115. Wein W, Roper B, Navab N. Integrating Diagnostic B-Mode Ultrasonography Into CT-Based Radiation Treatment Planning. IEEE Trans Med Imaging (2007) 26:866-79. doi: 10.1109/TMI.2007.895483

116. Coles CE, Cash CJC, Treece GM, Miller FNAC, Hoole ACF, Gee AH, et al. High Definition Three-Dimensional Ultrasound to Localise the Tumour Bed: A Breast Radiotherapy Planning Study. Radiother Oncol (2007) 84:233-41. doi: 10.1016/j.radonc.2007.06.016

117. Grimwood A, McNair HA, O'Shea TP, Gilroy S, Thomas K, Bamber JC, et al. In Vivo Validation of Elekta's Clarity Autoscan for Ultrasound-Based Intrafraction Motion Estimation of the Prostate During Radiation Therapy. Int J Radiat Oncol Biol Phys (2018) 102:912-21. doi: 10.1016/ j.ijrobp.2018.04.008

118. Modern Applications of 3D/4D Ultrasound Imaging in Radiotherapy. E Harris, D Fontanarosa, S Camps, F Verhaegen, editors. Bristol, UK: IOP Publishing (2021).

119. Octorina Dewi DE, Mohd. Fadzil M, Mohd. Faudzi AA, Supriyanto E, Lai KW. Position Tracking Systems for Ultrasound Imaging: A Survey. In: KW Lai, DE Octorina Dewi, Editors. Medical Imaging Technology: Reviews and Computational Applications. Singapore: Springer Singapore (2015) pp. 5789. doi: 10.1007/978-981-287-540-2_3

120. Bray FN, Simmons BJ, Wolfson AH, Nouri K. Acute and Chronic Cutaneous Reactions to Ionizing Radiation Therapy. Dermatol Ther (Heidelb) (2016) 6:185-206. doi: 10.1007/s13555-016-0120-y

121. Huang CJ, Hou MF, Luo KH, Wei SY, Huang MY, Su SJ, et al. RTOG, CTCAE and WHO Criteria for Acute Radiation Dermatitis Correlate With Cutaneous Blood Flow Measurements. Breast (2015) 24:230-6. doi: 10.1016/ j.breast.2015.01.008

122. Jang WH, Shim S, Wang T, Yoon Y, Jang WS, Myung JK, et al. In Vivo Characterization of Early-Stage Radiation Skin Injury in a Mouse Model by Two-Photon Microscopy. Sci Rep (2016) 6:19216. doi: 10.1038/srep19216
123. Yohan D, Kim A, Korpela E, Liu S, Niu C, Wilson BC, et al. Quantitative Monitoring of Radiation Induced Skin Toxicities in Nude Mice Using Optical Biomarkers Measured From Diffuse Optical Reflectance Spectroscopy. BioMed Opt Express (2014) 5:1309-20. doi: 10.1364/ BOE.5.001309

124. Chin LCL, Cook EK, Yohan D, Kim A, Niu C, Wilson BC, et al. Early Biomarker for Radiation-Induced Wounds: Day One Post-Irradiation Assessment Using Hemoglobin Concentration Measured From Diffuse Optical Reflectance Spectroscopy. BioMed Opt Express (2017) 8:1682-8. doi: 10.1364/BOE.8.001682

125. Diaz PM, Jenkins SV, Alhallak K, Semeniak D, Griffin RJ, Dings RPM, et al. Quantitative Diffuse Reflectance Spectroscopy of Short-Term Changes in Tumor Oxygenation After Radiation in a Matched Model of Radiation Resistance. BioMed Opt Express (2018) 9:3794-804. doi: 10.1364/ BOE.9.003794

126. Liu T, Zhou J, Yoshida EJ, Woodhouse SA, Schiff PB, Wang TJ, et al. Quantitative Ultrasonic Evaluation of Radiation-Induced Late Tissue Toxicity: Pilot Study of Breast Cancer Radiotherapy. Int J Radiat Oncol Biol Phys (2010) 78:811-20. doi: 10.1016/j.ijrobp.2009.08.071

127. Dean-Ben XL, Razansky D. Optoacoustic Imaging of the Skin. Exp Dermatol (2021) 30(11):1598-609. doi: 10.1111/exd.14386

128. Omar M, Aguirre J, Ntziachristos V. Optoacoustic Mesoscopy for Biomedicine. Nat Biomed Eng (2019) 3:354-70. doi: 10.1038/s41551-0190377-4

129. Cox JD, Stetz J, Pajak TF. Toxicity Criteria of the Radiation Therapy Oncology Group (RTOG) and the European Organization for Research and Treatment of Cancer (EORTC). Int J Radiat Oncol Biol Phys (1995) 31:1341-6. doi: 10.1016/0360-3016(95)00060-C

130. Hysi E, He X, Fadhel MN, Zhang T, Krizova A, Ordon M, et al. Photoacoustic Imaging of Kidney Fibrosis for Assessing Pretransplant Organ Quality. JCI Insight (2020) 5(10):e136995. doi: 10.1172/ jci.insight. 136995

131. van den Berg PJ, Bansal R, Daoudi K, Steenbergen W, Prakash J. Preclinical Detection of Liver Fibrosis Using Dual-Modality Photoacoustic/Ultrasound System. BioMed Opt Express (2016) 7:5081-91. doi: 10.1364/BOE.7.005081

132. Ida T, Iwazaki H, Kawaguchi $Y$, Kawauchi S, Ohkura T, Iwaya K, et al. Burn Depth Assessments by Photoacoustic Imaging and Laser Doppler Imaging. Wound Repair Regener (2016) 24:349-55. doi: 10.1111/wrr.12374

133. Hariri A, Chen F, Moore C, Jokerst JV. Noninvasive Staging of Pressure Ulcers Using Photoacoustic Imaging. Wound Repair Regener (2019) 27:48896. doi: 10.1111/wrr.12751

134. Rebling J, Ben-Yehuda Greenwald M, Wietecha M, Werner S, Razansky D. Long-Term Imaging of Wound Angiogenesis With Large Scale Optoacoustic Microscopy. Adv Sci (Weinh) (2021) 8:2004226. doi: 10.1002/ advs. 202004226

135. Aguirre J, Schwarz M, Garzorz N, Omar M, Buehler A, Eyerich K, et al. Precision Assessment of Label-Free Psoriasis Biomarkers With UltraBroadband Optoacoustic Mesoscopy. Nat Biomed Eng (2017) 1:0068. doi: 10.1038/s41551-017-0068

136. Regensburger AP, Brown E, Kronke G, Waldner MJ, Knieling F. Optoacoustic Imaging in Inflammation. Biomedicines (2021) 9(5):483-500. doi: 10.3390/biomedicines 9050483

137. Else TR, Hacker L, Bohndiek SE. Evaluating the Impact of Melanin on Spectral Colouring in Photoacoustic Imaging. SPIE Photons Plus Ultrasound: Imaging Sens (2021) 11642:116424M. doi: 10.1117/12.2577257

138. Sjoding MW, Dickson RP, Iwashyna TJ, Gay SE, Valley TS. Racial Bias in Pulse Oximetry Measurement. N Engl J Med (2020) 383:2477-8. doi: 10.1056/NEJMc2029240

139. Li X, Dinish US, Aguirre J, Bi R, Dev K, Attia ABE, et al. Optoacoustic Mesoscopy Analysis and Quantitative Estimation of Specific Imaging Metrics in Fitzpatrick Skin Phototypes II to V. J Biophotonics (2019) 12: e201800442. doi: 10.1002/jbio.201800442

140. Lui EY, Steinman AH, Cobbold RS, Johnston KW. Human Factors as a Source of Error in Peak Doppler Velocity Measurement. J Vasc Surg (2005) 42:972-9. doi: 10.1016/j.jvs.2005.07.014

141. Lai PK, Wang YA, Welsh AW. Reproducibility of Regional Placental Vascularity/Perfusion Measurement Using 3D Power Doppler. Ultrasound Obstet Gynecol (2010) 36:202-9. doi: 10.1002/uog.7608 
142. Nyayapathi N, Zhang H, Zheng E, Nagarajan S, Bonaccio E, Takabe K, et al. Photoacoustic Dual-Scan Mammoscope: Results From 38 Patients. BioMed Opt Express (2021) 12:2054-63. doi: 10.1364/BOE.420679

143. Freislederer P, Kugele M, Ollers M, Swinnen A, Sauer TO, Bert C, et al. Recent Advanced in Surface Guided Radiation Therapy. Radiat Oncol (2020) 15:187. doi: 10.1186/s13014-020-01629-w

144. O'Kelly D, Zhou H, Mason RP. Tomographic Breathing Detection: A Method to Noninvasively Assess In Situ Respiratory Dynamics. J BioMed Opt (2018) 23:1-6. doi: 10.1117/1.JBO.23.5.056011

145. Regensburger AP, Fonteyne LM, Jüngert J, Wagner AL, Gerhalter T, Nagel AM, et al. Detection of Collagens by Multispectral Optoacoustic Tomography as an Imaging Biomarker for Duchenne Muscular Dystrophy. Nat Med (2019) 25:1905-15. doi: 10.1038/s41591-0190669-y

146. Bohndiek S. Addressing Photoacoustics Standards. Nat Photonics (2019) 13:298-8. doi: 10.1038/s41566-019-0417-3

147. Bohndiek SE, Cox B, Joseph J, Gröhl J, Hacker L, Morscher S, et al. (2020). IPASC: A Community-Driven Consensus-Based Initiative Towards Standardisation in Photoacoustic Imaging, in: 2020 IEEE International Ultrasonics Symposium (IUS). pp. 1-4.

148. Press RH, Shu HG, Shim H, Mountz JM, Kurland BF, Wahl RL, et al. The Use of Quantitative Imaging in Radiation Oncology: A Quantitative Imaging Network (QIN) Perspective. Int J Radiat Oncol Biol Phys (2018) 102:121935. doi: 10.1016/j.ijrobp.2018.06.023

149. Sullivan DC, Obuchowski NA, Kessler LG, Raunig DL, Gatsonis C, Huang EP, et al. Metrology Standards for Quantitative Imaging Biomarkers. Radiology (2015) 277:813-25. doi: 10.1148/radiol.2015142202
150. O'Connor JPB, Aboagye EO, Adams JE, Aerts HJWL, Barrington SF, Beer AJ, et al. Imaging Biomarker Roadmap for Cancer Studies. Nat Rev Clin Oncol (2017) 14:169-86. doi: 10.1038/nrclinonc.2016.162

Conflict of Interest: SB has previously received research funding from PreXion Corporation, which (Photoacoustic imaging division) was later acquired by CYBERDYNE Inc. and research support from iThera Medical GmbH, both vendors of photoacoustic imaging equipment. MT would like to disclose that he is currently employed at Merck \& Co.

The remaining authors declare that the research was conducted in the absence of any commercial or financial relationships that could be construed as a potential conflict of interest.

Publisher's Note: All claims expressed in this article are solely those of the authors and do not necessarily represent those of their affiliated organizations, or those of the publisher, the editors and the reviewers. Any product that may be evaluated in this article, or claim that may be made by its manufacturer, is not guaranteed or endorsed by the publisher.

Copyright (c) 2022 Lefebvre, Brown, Hacker, Else, Oraiopoulou, Tomaszewski, Jena and Bohndiek. This is an open-access article distributed under the terms of the Creative Commons Attribution License (CC BY). The use, distribution or reproduction in other forums is permitted, provided the original author $(s)$ and the copyright owner(s) are credited and that the original publication in this journal is cited, in accordance with accepted academic practice. No use, distribution or reproduction is permitted which does not comply with these terms. 